\title{
Application of Information Technology in Comprehensive Reform of Agricultural Water Price in Irrigated District
}

\author{
Yongtao Wang ${ }^{1}$, Wei Huang ${ }^{1}$, Li Liang ${ }^{1}$, Yuewei Chen ${ }^{2}$ and Zhongfei Zhou ${ }^{2}$ \\ ${ }^{1}$ Water Resources Research Institute of Guizhou Province, Guiyang, 550002, China \\ ${ }^{2}$ Guizhou Dongfeng Automation Technology Co., Ltd., Guiyang, 550002, China
}

\begin{abstract}
This paper studies the application of information technology in comprehensive reform of agricultural water price in irrigated area. Firstly, the requirement of the system is analyzed, the scheme and the structure of the information system is put forward. Secondly, the following technology are studied : The open channel flow measurement, automatic gate opening and closing control, video monitoring, automatic irrigation control technology based on moisture monitoring, and water price management of RF IC card. The software development environment is Visual Studio2015, and the database adopts a structured design method. Finally, the system management and the RF IC card control software are designed, which can be applied to the comprehensive reform of agricultural water price in large and medium-sized irrigated area, and effectively enhance the intelligent level of irrigation water management in irrigated area.
\end{abstract}

Keywords-information technology; comprehensive reform of agricultural water price in irrigated area; system design and application

\section{INTRODUCTION}

The core of the information construction of the comprehensive reform of agricultural water price in irrigated area is the accurate measurement of the agricultural water use, realizing an open, fair and just water price and water fee collection mechanism. It can eliminate the serious waste problem caused by the inaccurate measurement of water, and it can save water effectively. The effective measurement and application of agricultural irrigation water can greatly reduce the cost of agricultural water management. At present, the measurement of water fee in Guizhou province is distorted, and the charge mode mainly adopts the settlement by acres of flat, it causes many problems, such as separation of water fee and water consumption, occurrence of water grabbing, waste of water resources, and frequent disputes of charge, even though, an outstanding problem came about that the water conservancy facilities are being damaged. The information construction in irrigation area can achieve an effective combination of accurate measurement and quantitative irrigation. It can solve the difficulty of collecting water fee through pre charging, at the same time, the corresponding water consumption and water fee can be realized through the regulation of rate. It can also improve the consciousness of the masses in water saving, solve the bottleneck management and charge problems of agricultural irrigation water from the technical level. In the comprehensive reform of agricultural water price in irrigation area, the application of information is of great significance in raising awareness of water saving and closing relationship between cadres and masses.

\section{EASE OF USE}

\section{A. Scheme Design of the System}

According to the characteristics of the fragmentation, fragmentary and undulating features of the arable in the irrigation area of Guizhou mountain area, the construction of information system in comprehensive agricultural water price reform based on the core of the field network is implemented. It can solve the mismanagement caused by the numerous project sites and the wide range. To build an integrated control system which assembles remote data acquisition, remote video transmission, remote monitoring control, automatic gate opening and closing, credit card charging system and data center.

\section{B. Maintaining the Integrity of the Specifications}

In the irrigation area, the water intake of the reservoir is equipped with water metering facilities to achieve the control of the total water intake. At the same time, we set up video monitoring system, automatic gate opening and closing, automatic irrigation control and IC card charging system. Through building remote telemetry station, data acquisition, transmission and application will be realized, and the information monitoring system of the comprehensive reform of agricultural water price will be built up step by step. It can effectively manage and maintain information about water exploitation, water fee collection, archives and so on. To achieve information sharing, interconnection, so the water using parties can know the cost of water in a timely, accurate and comprehensive way. To achieve efficient and precise management of agricultural water and fertilizer, improve quality and increase efficiency. At the same time, it can improve the agricultural water consumption and the social benefits of new rural construction, and achieve comprehensive benefits such as water saving and emission reduction.

\section{TECHNICAL SCHEMES}

\section{A. Open Channel Flow Measurement Technology}

The flow monitoring in the open channel is indirect measurement, which cannot be measured directly, but to measure the water level, the water depth, the starting distance of the cross section, the velocity and so on, and then calculate 
the flow by mathematical model. Therefore, velocity, water level, water depth and starting distance become direct monitoring elements of water resources. A pipe flow meter for full pipe is used to directly measure the flow data. A pipe flow measuring facility for a non-full pipe which is also an indirect measurement is used to measure water level and velocity, then we can get the flow through mathematical model. The purpose of water intake monitoring in the irrigation area is to get the amount of water flowing through the open channel or pipeline survey section in a certain period of time, that is, the total amount of water measured by volume (cumulative water). To get the total water volume, we also need to measure the process of flow changing with time.

\section{B. Flow Measurement Method}

According to the actual situation that there are a large volume of sediment in water in the project area, we use ultrasonic Doppler open channel flow meter to measure the flow rate, and then convert it into flow measurement. The flow meter mainly consists of two parts, the sensor and the main engine. The sensor contains two transducers for transmitting and receiving. When the sensor is in the same direction with the current, namely the counter current condition is right: (1) the frequency of the receiving sensor is the same as that of the transmitting frequency, which indicates that the speed of the water flow is equal; (2) the former is greater than the latter, and the faster the frequency, the faster the water speed is; (3) the former is lower than the latter, the slower the frequency of the transducer is, the slower the water speed is. With this principle, flow velocity and direction of flow can be calculated by hardware and software. Select the type: the split type-XIHU-Field Irrigation Doppler Flow meter, a type of online measurement. General sensor output is 485 signals, includes speed and flow data.

\section{Automatic Gate Opening and Closing Control Technology}

With the design principle of "unattended and few people on duty", the automatic control system of the hand power gate is designed to develop an automatic in-plant or remote control system for the user, which can be developed by the integrated application of sensing technology, automation control technology, computer software and hardware technology, network communication technology and so on.

The gate control system mainly realizes the automatic opening and closing of the dam surface according to the river management, flood control, the landscape water level-the flow requirement through the detection of the water level in the upper reaches of the gate. It can automatically adjust the state: opening or closing and the opening degree according to the change of the water level, to ensure the safety of the river. It can also realize remote control in the monitoring center, or automatic control, and real-time image information collection and transmission. The system can display the water level, flow, video picture and so on and the sluice control system in a software picture, making the remote operation more intuitive, reaching the goal of unmanned and scientific scheduling.

The front-end sensor will monitor the state information, such as the level, the height and the image of the automatic gate, the water situation and so on, then the control terminal collect and process these information, all kinds of information will be transmitted to the information software platform through the network transmission of wired or wireless network. Managers can monitor and control in real time in the monitor center to ensure safe and reliable operation of gates, It can save manpower and material resources, and realize efficient and convenient management. The transmission of hypertext information, such as image and video, is transmitted by optical fiber, and other data information of water level, flow, height and so on is transmitted by GPRS wireless transmission. The system is powered by the city power supply.

\section{Video Monitor Technology}

The outdoor dome camera are arranged in the key monitoring points, such as, inside and outside of the storage machine room of the management center, upstream and downstream of the river, so as to realize the omnidirectional monitoring and management of the scene around the upstream and downstream gate and the situation of automatic. Video information collected in real time can be stored in computers. As historical data, it has very important value for accident analysis and responsibility investigation.

Video monitor system consists of four parts: front-end device, transmission device, control device, record and monitoring device. The front-end equipment is composed of high resolution color cameras installed at monitoring points, omnidirectional cloud platforms, zoom lenses and outdoor professional protective devices. It is mainly responsible for image data acquisition and signal processing. Transmission devices choose different transmission modes according to transmission distance and image quality requirements, we need to consider whether or not we want to compress the video signal in the actual implementation according to the bandwidth of the communication network of the management center.

The centralized control device is responsible for the control of front-end device and image switching, omnidirectional cloud platform and three dimensional variable lens control. It can also control partitioned and synchronize the monitoring image, and also provide image retrieval and processing function by software.

\section{Scheme design}

Outdoor dome camera are arranged in the management center and field irrigation areas to monitor and manage the surrounding scene in all directions. The real-time collection of video information can be stored in the local monitoring terminal computer. As a historical data, it is of great value for accident analysis and responsibility investigation.

The front end device of the local monitoring terminal is composed of cameras and display devices. The camera is responsible for the acquisition of image and data, the control of the cloud platform and the processing of the image signal. The display device uses a liquid crystal display for video image display. The camera can rotate in a full range of 360 degrees, 90 degrees vertically, its lens can focus twelve times, zoom and adjusting the size of the aperture. So the general and local key observation of the general situation in the $300 \mathrm{~m}$ range of the monitoring area can be realized in the case of no occlusion. The staff can know the monitoring site information by observing the video displayed. 


\section{E. The Integration of Irrigation and Fertilization Technology}

The automatic irrigation and fertilization control system based on soil moisture monitoring is an important content of the precise management of water and fertilizer in the comprehensive reform of agricultural water price. Through moisture monitoring and EC/PH monitoring of soil, the adaptive irrigation and fertilization according to the different growth period of crops is realized. In the field of irrigation and fertilization, the fuzzy intelligent control model is adopted to realize the data analysis and dynamic management of the crop growth process, and the application demonstration is carried out. The system has high integration degree, strong expansion performance, stable, easy and convenient operation.

The system is mainly composed of water supply, sand and gravel filter, water storage tank, constant voltage variable frequency water supply, fertilizer applicator, field irrigation valve, fertilizer storage tank, and transportation pipeline and computer control system. It has the advantages of $60 \%$ water saving, 50\% fertilizer saving, 30\% increase production, environmental protection and manpower saving.

The fertilizer applicator mainly consists of motor pump, fertilizer, mixing device, filter, EC/PH monitoring and control feedback device, pressure difference constant device, automatic control system and so on. According to the input conditions or soil moisture, evaporation, rainfall and light intensity, it can adjust and control irrigation and fertilization automatically. In the process of fertilization, we can select and set up the irrigation and fertilization program, and make automatic timing and quantitative irrigation and fertilization according to the established procedures.

The system monitors soil's real-time nutrient status through soil nutrient sensors, and completes the Precise Fertilization function by fertilizing valve and fertilizing tank. When the water content is constant, the urea content in the coupling fluid increases, the concentration of coupling fluid will also increase correspondingly. At this time, the concentration of $\mathrm{N}$ element increases and the EC value also increases. Therefore, the size of EC can reflect the concentration of $\mathrm{N}$ and the content of urea. The coupled water and fertilizer model is [5]:

$$
Q=\frac{0.46 b(1-x 1)}{x 1}=\frac{0.46 b \times\left(\sqrt{\frac{184.89-y 1}{0.2598}}-8.97\right)}{9.97-\sqrt{\frac{184.89}{0.2598}+99.4}}
$$

\section{F. 3.5 Radio Frequency IC Card Technology}

At present, there are many kinds of products based on RFID technology, but they are not compatible with each other. Radio frequency IC card can complete the communication between equipment through radio wave. In this application, RF IC card is the key link between host computer and slave computer, and is the key to intelligent control. According to the different requirements, the system set up five kinds of IC card that is user card setting card, management card, clearing card and transfer card.
The function of IC card reader is that it has the function of limiting water intake, and will stop the pump automatically when the annual cumulative water consumption exceeds the set limit. It also has lightning protection function to ensure the safe operation of the control terminal in thunderstorm season. The handset has the function of wireless meter reading. It can copy all the farmers' water records in the village within a range of 20 meters around the key. The IC cards in different villages cannot be used with each other, that is, a village card cannot be used in another village. When the power is outrage, the data of the irrigation control box will not be lost. After the electricity come, the card can continue to use.

An IC card irrigation control box can be used by many farmers. A farmer can also use several IC card irrigation control boxes. When the amount of the card is less than the set lower limit, the IC card irrigation control box will send out the alarm information automatically, prompting farmers to recharge in time.

\subsubsection{RF circuit design}

The essence of the RF circuit is an LC oscillator.MF_RC500 is a highly read and write card chip that integrates ISO14443A protocol. It is widely applied in the field of con-tactless communication. The serial chips take advantage of the advanced modulation and demodulation concept and fully integrates all kinds of passive and non-contact communication methods and protocols under 13.56MHz.It supports multi-layer applications of ISO14443A.The internal transmitter part can directly drive near operating distance antenna (up to $10 \mathrm{~cm}$ ) without increasing active circuit. The receiver part provides a robust and effective demodulation and decoding circuit, which is used to process transponder signals compatible with ISO14443A.The digital part processes ISO14443A frames and detect errors(parity CRC). D0-D7 is the multiplex bus for address and data. ALE implements address latch. NRD and NWD are read and write gated signals respectively, RX receives $12.56 \mathrm{MHzASK}$ modulation signal. L1, C3 and L2, C4 filter high order harmonics in signal. $\mathrm{L} 1=\mathrm{L} 2=2.2 \mu \mathrm{F}$, $\mathrm{C} 3=\mathrm{C} 4=47 \mu \mathrm{F}$, The cut-off frequency $f c$ $=15.65 \mathrm{MHz}$ of the filter can be calculated by formula (1), which is higher than that of the reader's $13.56 \mathrm{Mhz}$, meeting the frequency requirement.

$$
f c=\frac{1}{2 \pi \sqrt{\mathrm{LC}}}=\frac{1}{2 \pi \sqrt{2.2 \mu \mathrm{H} \times 47 \mathrm{PF}}}=15.65 \mathrm{MHz}
$$

\section{USING THE TEMPLATE}

\section{A. Information Management System}

According to the structured design method, the data items and data structures mainly contained in the data dictionary are shown below: (1) Administrator information table, include name, phone, category, password, job number and so on. (2)Users information table, include number, name, address, phone, ID card number, account opening time, remark. (3) Water purchase information table, include: user number, water meter number, purchase quantity, last balance, current balance, purchase date and administrator number. (4) Village 
information table, include: user town name, address, number of users, village head's name and telephone, and so on. (5) Water meter information table, include: user town name, water meter type, installation time, address, village head's name and telephone, and so on. (6) Water fee information table, include: water meter type, installation time, user town name, village head's name, telephone number, rate and so on.

In SQL Server2005, the Smart IC cards irrigation management database is created to define the above 6 tables, for example, the user's water purchase information table is designed as shown in Table 1.

TABLE I. USER'S WATER PURCHASE INFORMATION TABLE

\begin{tabular}{|c|c|c|c|c|}
\hline Column name & $\begin{array}{l}\text { Data } \\
\text { type }\end{array}$ & $\begin{array}{c}\text { Lengt } \\
\mathrm{h}\end{array}$ & $\begin{array}{c}\text { NULL or } \\
\text { NOT }\end{array}$ & Remarks \\
\hline User ID & nvarchar & 20 & $\begin{array}{l}\text { NOT } \\
\text { NULL }\end{array}$ & $\begin{array}{c}\text { User } \\
\text { number }\end{array}$ \\
\hline Watermeter ID & int & 5 & NULL & $\begin{array}{l}\text { Meter } \\
\text { number }\end{array}$ \\
\hline Purchase & real & 5 & NULL & $\begin{array}{l}\text { Purchase } \\
\text { quantity }\end{array}$ \\
\hline Former Money & real & 5 & NULL & $\begin{array}{c}\text { Last } \\
\text { balance }\end{array}$ \\
\hline Current Money & real & 5 & NULL & $\begin{array}{l}\text { Current } \\
\text { balance }\end{array}$ \\
\hline Purchase Date & $\begin{array}{l}\text { Date } \\
\text { time }\end{array}$ & 10 & NULL & $\begin{array}{c}\text { Purchase } \\
\text { date }\end{array}$ \\
\hline $\begin{array}{l}\text { Administrator } \\
\text { ID }\end{array}$ & int & 5 & $\begin{array}{l}\text { NOT } \\
\text { NULL }\end{array}$ & $\begin{array}{l}\text { Administrat } \\
\text { or number }\end{array}$ \\
\hline
\end{tabular}

\section{B. Design and Implementation of System Software}

The management system mainly completes the management of IC card, business statistics, and business management and information maintenance. The system software is developed by Visual Studio 2015 an integrated development environment, the development language is C\#, and the database is SQL Server 2015.Information maintenance mainly includes water fee type management, town information management, user's type management, water meter information management, tax and fee management, etc. The main business of IC card management include handle user's card, cancel the account, recharge, report the loss etc. The system statistics mainly completes the statistics of water consumption and water charges. The system management mainly includes user login information management, privilege management, password management, security management and storage of corresponding operation logs.

\section{Software Function of Water Station}

(1) Real-time monitoring: supports GIS map form, list form, and icon form which can realize real-time online monitoring, it's easy to grasp every state; (2)Water rights management: with the goal of "total quantity control and quota management", we build three levels of water right distribution and trading from top to bottom: water rights distribution, transaction, and village, to realize the market management of water rights; (3) Assets management of irrigation system, records assets attribute, purchase and maintenance records in detail, for inquiring assets information at any time; (4) Automatic generation of various reports, such as water use, year, month, daily tables of electricity consumption, various forms analysis and display analysis and data after digging, it can also assists in the regional agricultural water intake plan; (5) It supports remote configuration and intervention control of each controller, it needs to enter the operation code to open and close the pump; (6) Statistical analysis: includes alarm query, operation query, water consumption query and recharge query. At the same time, it can print historical records at any time; (7) Information release: supports for adding, deleting, searching and modifying water users' information, price information and irrigation crop types; (8) System management: user security management, it supports historical operation records query, responsibility tracing; (9) Alarm inquiry: It supports device failure automatic alarm, the alarm mode, including short message, APP and PC software alarm.

\section{Design of Intelligent IC Card Control Software}

Define interrupt 0 as interrupt things for effective card, interrupt 1 as serial communication interruption function system, and expand to remote automatic meter reading function. After the power reset, the MCU enters the initialization state, it will scan the external interruption of the system: 0 and 1 . When the effective brush card is scanned, the interrupt 0 is effective; the MCU reads and writes IC card information. If the IC card information shows that the remaining amount of water is sufficient, and it is proved to be the legal user, the valve will be opened, the irrigation action is performed, the water flow is measured by the flow meter at the same time. The corresponding water treatment is taken. If the user wants to finish the irrigation operation immediately, it needs to swipe card again, and the system will turn off the valve automatically. If the IC card information shows that the remaining amount of water is insufficient or 0 , and the current user is illegal, the system starts to alarm and closes the solenoid valve, and the system is in standby state. The software development of control system adopts uVision4, an integrated development environment. The programming language uses Keil C51.

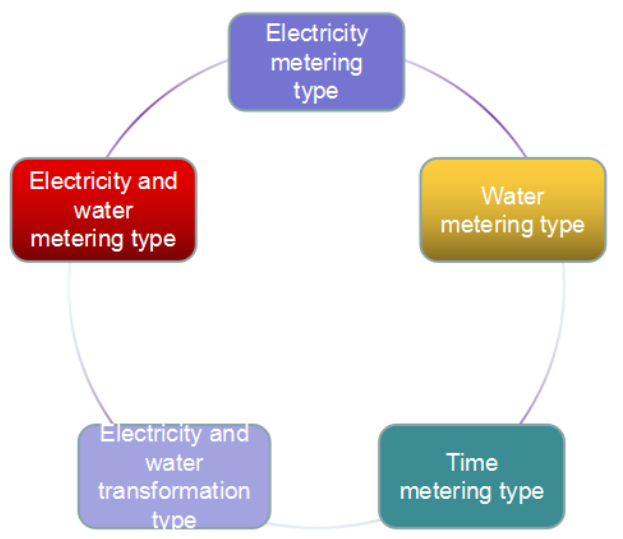

FIGURE I. SCHEMATIC DIAGRAM OF MEASUREMENT MODE

\section{CONCLUSION}

(1) The system can achieve effective combination of accurate measurement of agricultural irrigation water and quantitative irrigation. Through pre-charge, it can solve the difficulties of collecting taxes and fees. At the same time, the corresponding water consumption and water cost can be 
achieved through rate adjustment. It can improve the consciousness of water saving of the masses, solve the bottleneck problem of agricultural irrigation water management mode and the difficult water charge from the technical level. Adapting to the application and popularization in large and medium-sized irrigation districts, we can improve the level of intelligent management of agricultural irrigation water.

(2) Through monitoring, transmission, diagnosis, decision-making and water dynamic management of crops, the system centered on promoting the efficiency of agricultural water saving and improving the formation mechanism of agricultural water price, and implementing the systematic project solution of "water" as the core of the system from water source to water user terminal. Comprehensive automatic control and information management is achieved in terms of sluice automatic opening and closing, credit card prepayment, automatic irrigation and water volume monitoring. In the light of local conditions, the comprehensive benefit of the project area is obviously improved, and a comprehensive reform model can be formed to promote the comprehensive reform of agricultural water price and lay the foundation for the comprehensive reform of agricultural water price.

\section{ACKNOWLEDGMENT}

This work was financially supported by the Project of Science and Technology Department in Guizhou "Integrated service enterprise of modern efficient water-saving irrigation technology action plan in Guizhou mountain area” Subject number: The platform talented people of Guizhou [2016]5717. Project of Science and Technology Department in Guizhou "Development and demonstration of characteristic and efficient agricultural water and fertilizer precision intelligent control system in modern irrigation area of Guizhou" The platform talented people of Guizhou [2017]2596. Research project of Water Resources Department of Guizhou Province "Development and demonstration of intelligent water saving irrigation technology in modern irrigation area of Guizhou"; "Research on collection and treatment technology mode of rural domestic sewage in Guizhou”.

\section{REFERENCES}

[1] Jiang Jing, Zhang Xuesong. Design of Contactless Intelligent Water Meter Based on AT89S52. [J]. Journal of North China Institute of Science and Technology. 2012.1(9):79-81.

[2] Wang Kewu, Zheng Wengang, Shen Changjun. Design of Pre-payment Water Meter for Random Irrigation Based on RF IC card.[J]. Water Saving Irrigation. 2010.12:67-68.

[3] Zhou Xuelei. The Application Study of Water Metering and Dispatching Management System of Facility Agriculture.[D]. Taiyuan University of Technology. 2011,5:21-23.

[4] Wang Yongxi. Design and Develop of the Control System for Water-Saving Irrigation Based on the Radio Frequency IC Card.[D]. Lanzhou University. 2010,11:21-24.

[5] Zeng Xiaoping, Ren Jiayu. Design of Contactless IC Card Interrogator Based on RFID.[J]. Computer Measurement \& Control. 2010.18(10):2357-2359. 